\title{
La Psicología Educacional y el sistema de educación en Cuba
}

\author{
Guillermo Arias Beatón
}

\begin{abstract}
Resumen
Este trabajo incluye un análisis del papel de la Psicología Educacional en el desarrollo y organización del Sistema Nacional de Educación cubano. Se mostrará la evolución del pensamiento filosófico, psicológico y pedagógico desde el siglo XVIII hasta nuestros días y cómo se ha enriquecido con el debate que exige la necesidad de una aplicación práctica de los contenidos teóricos y de las investigaciones empíricas en el perfeccionamiento de la Educación de un pueblo, impulsados por la concepción de que las personas pueden y deben apropiarse de los contenidos de la educación, como condición para promover el desarrollo sostenido de un proyecto social y cultural revolucionario. La necesidad de realizar estudios y sistematizaciones críticas de los contenidos teóricos, metodológicos y empíricos de toda la psicología, para construir un sistema de Educación que garantice la calidad necesaria para que se logre el aprendizaje de todos los escolares con su diversidad. La importancia que tiene para una lucha contra el colonialismo cultural, que la psicología y los psicólogos, en conjunto con las demás ciencias afines a la Educación, participen en el debate científico y técnico y de base a las políticas públicas sobre la Educación y cómo, al incidir aun más, en el perfeccionamiento de esta políticas, hará mas significativo, el papel de la Psicología con el compromiso social y cultural del país y sobre todo con el ser humano.
\end{abstract}

Palabras claves: educación, aprendizaje, desarrollo, psicología escolar.

\section{Educational Psychology and the educational system in Cuba}

\begin{abstract}
In this work we present an analysis of the role of Educational Psychology and the organization of the Educational system in Cuba. We propose to show the evolution of the philosophical, psychological and pedagogical ideas from the XVIII century to this date. We argue that this field of study has been enriched through debates that require a practical application of the theories and of empirical research in the improvement of the contents in Education as a condition to promote the development supported by a social and cultural revolutionary project. We need to accomplish studies and critical formulations on the methodological, theoretical and empirical contents in Psychology. This will help us to build a system of Education that grant a quality necessary to promote the learning to all school children in their diversity. Psychology, together with Education has a very important role in the struggle against cultural colonialism, so it is important to promote scientific debates, in this area always concerning with the social and cultural commitment to the country.
\end{abstract}

Keywords: Education, learning, development, school psychology.

\section{A Psicologia Educacional e o sistema de educação em Cuba}

\begin{abstract}
Resumo
Este artigo apresenta a análise do papel da Psicologia Educacional no desenvolvimento e organização do Sistema Nacional de Educação em Cuba. Destacar-se-á a evolução do pensamento filosófico, psicológico e pedagógico, desde o século XVIII até nossos dias, e como se enriqueceu o debate que exige a necessidade de uma aplicação prática dos conteúdos teóricos e das pesquisas empíricas para o aperfeiçoamento da Educação de um povo, impulsionados pela concepção de que as pessoas podem e devem apropriar-se dos conteúdos da educação como condição para promover o desenvolvimento sustentado em um projeto social e cultural revolucionário. A necessidade de realizar estudos e sistematizações críticas dos conceitos teóricos, metodológicos e empíricos de toda a psicologia, para construir um sistema de Educação que garanta a qualidade necessária para que se atinja o objetivo de aprendizagem de todos os escolares com sua diversidade. A importância que possui para uma luta contra o colonialismo cultural, que a psicologia e os psicólogos, em conjunto com as demais ciências afins à Educação, participem do debate científico e técnico, construindo a base para as políticas públicas sobre a educação e como, ao se inserir ainda mais no aperfeiçoamento destas políticas, tornará ainda mais significativo o papel da Psicologia com o compromisso social e cultural do país e sobretudo com o ser humano.
\end{abstract}

Palavras-chave: educação, aprendizagem, desenvolvimento, políticas públicas, psicologia educacional. 


\section{Introductión}

La Psicología Educacional ha desempeñado, durante estos últimos cuarenta años, un papel significativo en el desarrollo y organización del Sistema Nacional de Educación Cubano y en los logros de la Educación de nuestro pueblo. Su presencia ha sido indiscutible, en el debate continuo de su mejoramiento y en la erradicación de los errores y dificultades que naturalmente se han presentado y se presentan, dada la complejidad de cualquier proceso de planeamiento y puesta en prácticas de programas de una Educación para todos y de calidad. Todo se ha hecho a través de una labor colectiva, interdisciplinaria y comprometida social y culturalmente, produciendo también, sus políticos, especialistas y profesionales destacados (Congreso de Educación y Cultura, 1971; Ministerio de Educación, 1973, 1975).

Uno de los aspectos más significativos es que tanto los políticos, en nuestro período revolucionario, como los científicos y pensadores progresistas, han tenido en cuenta, interpretado y aplicado, el pensamiento filosófico, psicológico y pedagógico cubano desde el siglo XVIII hasta nuestros días.

También ha sido importante el compromiso de estos, con las exigencias y demandas del pueblo cubano, en relación con el papel de la educación, porque nuestra identidad como pueblo, nuestra nacionalidad, gracias a aquellos pensadores, surgió muy unida a la idea de que solo con una buena educación se puede lograr la formación y desarrollo de dicha identidad, de la conciencia nacional y de las ideas de libertad e independencia del coloniaje español o de cualesquiera de ellos, más tarde (Luz y Caballero, 1999, 2001a, 2001b; Martí, 1975, 1983; Varela, 1999). En este sentido, se presenta y materializa la idea optimista, de que todos los seres humanos son susceptibles de ser educados, pueden aprender y desarrollar su conciencia (Comenio, 1983; Luz y Caballero, 1999, 2001a, 2001b; Martí, 1975; Rousseau, 1973; Varela, 1999; Vivés, 1997;).

Ha estado presente, en ese pensamiento, que la educación debe ser, desde lo cultural propio o nacional, pero enriquecida por los conocimientos y la cultura de otros pueblos y pensadores del mundo. Con orgullo, hasta hoy, decimos que somos una confluencia e integración de culturas y conocimientos. Pero desde aquellos tiempos sabemos, que es necesario e imprescindible el instrumento metodológico de la crítica y la autocrítica sistemática del conocimiento, para lograr esa síntesis o integración y dejar de ser eclécticos (Arias, 1999a, 2002a, 2002d, 2004, 2005; Fariñas \& Arias, 2002; Luz y Caballero, 2001a, 2001b; Vygotski, 1991).

Aquellos pensadores nos enseñaron que todo conocimiento debe ser sometiendo a una crítica científica sistemática, todo lo que nos llega, antes de integrarlo a nuestro saber, debe ser considerado en sus ventajas y desventajas. Por eso hoy en día, desde la Cátedra L.S. Vygotski, los que debatimos y trabajamos en ella, postulamos la continuidad y el rescate de una sistematización crítica de la teoría del
Enfoque Histórico Cultural de todos los tiempos y uno de sus principales contenidos, es hacer un análisis profundo de las bases de nuestro pensamiento histórico cultural en nuestros pensadores de origen y de toda la América Latina (Arias, 1999a, 1999b, 2002a, 2002b, 2002d, 2004a, 2004b, 2005, 2006; Corral, 1999; Cruz-Tomás, 1995; Domínguez, 1995; Fariñas, 1999, 2005; Fariñas \& Arias, 2002; Fariñas \& Coelho, 2008; Febles Elejaldes, 1987, 1997, 2001; López e cols.,1990-2003; Serra, 2003; Valdés, 1984; Vygotski, 1972, 1981, 1987, 1988, 1989, 1991, 1993, 1995, 1996, 1999, 2001, 2004).

Creemos que no será difícil de observar las semejanzas que existen entre este referente teórico y el contenido del pensamiento progresista cubano de todos los tiempos (Arias, 2005; Serra, 2003; Valdés, 1984) y seguramente de América Latina, desde Sarmientos, Simon Rodríguez y Hostos hasta Freire.

Lo breve de esta intervención, no me permiten profundizar mucho más en esto, pero sí decir y exhortar a trabajar, en este importante aspecto, porque creo que hay enormes coincidencias y necesidades de hacerlo, para que nuestra educación se constituya, con bases propias, profundas y sólidas, en una educación para todos y de calidad.

Las coincidencias se pudieran resumir en: la necesidad de una educación para todos y que contribuya al proceso de formación y desarrollo humano, que el conocimiento es una dinámica entre lo sensorial, lo empírico y lo racional, la necesidad de educar en el amor y por el amor, la necesidad de ver el todo en su dinámica e interdeterminación con las partes, la necesidad de emplear diversos métodos en el proceso de construcción de los conocimientos y en el proceso de la educación, la necesidad tanto del papel activo del educador como del escolar, que el educador organice y dirija su trabajo para lograr la activa creación del escolar y el redescubrimiento del conocimiento, la relación entre la teoría y la práctica, entre otros importantes problemas (Arias, 2005, 2006; Betancourt \& González, 2003; Bozhovich, 1976; Davidov, 1991; Fariñas, 1995, 2004a, 2004b, 2005; Freire, 1971, 2001; Leontiev, 1966, 1981, 1991, 1997; López, 1990, 1991, 1992; Luz y Caballero, 1999, 2001a, 2001b; Martí, 1975; Mújina, 1981; Novoselova, 1981; Rubinstein, 1965, 1972; Varela, 1999; Varona \& Guevara, 1984; Venguer, 1976; Venguer \& López, 1990; Vygodskaia, 1997; Vygotski, 1981,1988, 1989, 1991, 1993, 1995, 1996, 1999, 2001;).

Por último, estamos convencidos que esto que referimos a Cuba, es una característica también de nuestra América, lo hemos estudiado y visto en México, Puerto Rico, Argentina, Brasil, Perú, Venezuela, Chile, entre otros.

Creo que un estudio que devele esto, es una de las vías efectivas para desarrollar el anticolonialismo y la no dependencia que promulgamos, eliminar la mentalidad de colonizados y promover una conciencia de independencia y autonomía, de crítica y autocrítica, que permita rechazar y no practicar, los hegemonismos, las imposiciones y la tendencia a la creación de ídolos que tanto daño hacen al conocimiento y su aplicación. Trabajar orientados por el 
respeto mutuo, la tolerancia, la comprensión, la solidaridad y el intercambio crítico de conocimientos.

La psicología educacional cubana, desde su organización y presencia sistemática en el Ministerio de Educación, los Dpto. de las Facultades de Psicología y las universidades Pedagógicas, los centros y Dpto. de las universidades en general y todos los psicólogos, psicopedagogos, pedagogos y educadores han contribuido significativamente al desarrollo de las políticas públicas en el período revolucionario.

No se puede hablar de ninguno de los logros de la educación en Cuba, donde no hayan estado presentes las investigaciones, los debates científicos, las experiencias prácticas y la reflexión crítica de estos profesionales vinculados al pensamiento y a la práctica de la psicología educativa.

Sin embargo, hay algo sumamente importante que se debe destacar y no se puede dejar de mencionar, la estrecha relación en este trabajo con, los políticos, otros profesionales y científicos, como sociólogos, lingüistas, médicos, matemáticos, biólogos, químicos, entre muchos otros.

La investigación científica, ha sido un instrumento esencial. No solo desarrollando investigaciones teóricas, experimentales, formativas y cualitativas, sino realizando aquellas que conducen más directamente a la introducción en la práctica, de los resultados de aquellas más teóricas, experimentales, formativas experimentales y descriptivas (Acosta, 1998; Ávila, 2002; Cordero, 2001; Cruz, 1998; Cuesta \& B. Hernández, 2000; Dávila, 2006; Días, 2005; Esteva Boronat, 2002; Febles Elejaldes, 1988; Figueredo, 1999; Fernández, 2003; Gómez, 1980, 1988, 1995; Gómez \& Borgato 1988; González, 2000; Gutiérrez, López, \& Arias, 1990; I. H. Pardo, 2005; J. Betancourt, 1999, 2002; J. M. G. Hernández, 2002; López, 1990, 1991, 1992; López \& Borgato, 2002; López \& Gómez, 1986; Lorenzo, 1980, 1981, 1982, 1987, 1988, 1990, 1995a, 1995b; Louis, 2003; M. del P. S. Rodríguez, 2001; Mendoza, 1988a, 1988b, 1989, 1990; MINED-CELEP, 1999; Pacheco, 1998, 2002; P. I. Pardo, 1995; Reyes, 2005; Rivero, 1999, 2001; Rivero \& Quílez, 1995a, 1995b, 2001; Roloff e cols., 1987; Ruiz, 2001; Sarduy, 1996a, 1996b; Sotomayor \& Muñoz, 1999; Tejeda, 2007; Tintorer, 1998; Tocabens, 2001; Velázquez, 2002; Y. A. Betancourt, 2005).

Aunque considero que ésta ha sido y es, una labor importante, se deberá ampliar para continuar mejorando, aun más, nuestra labor educativa y sobre todo nuestra labor y acervo científico y profesional. El debate profesional y científico sin dudas ha sido esencial, aunque también habrá que rescatarlo con nuevos bríos.

La psicología educacional cubana ha estado presente de forma significativa en: el desarrollo masivo y productivo de la educación inicial y preescolar desde sus inicios, en los logros del aprendizaje de la lectura y la escritura, el español, las matemáticas, la formación de valores, la formación de maestros y educadores en general, en el trabajo con las familias y lo relacionado con el imprescindible vínculo del hogar con la escuela y la labor de los medios de comunicación masiva como parte importante de la sociedad, para lograr una mejor educación de nuestros niños, niñas, adolescentes y jóvenes (Acosta, 1998; Ávila, 2002; Cordero, 2001; Cruz, 1998; Cuesta \& B. Hernández, 2000; Dávila, 2006; Días, 2005; Esteva Boronat, 2002; Febles Elejaldes, 1988; Figueredo, 1999; Fernández, 2003; Gómez, 1980, 1988, 1995; Gómez \& Borgato 1988; González, 2000; Gutiérrez, López, \& Arias, 1990; I. H. Pardo, 2005; J. Betancourt, 1999, 2002; J. M. G. Hernández, 2002; López, 1990, 1991, 1992; López \& Borgato, 2002; López \& Gómez, 1986; Lorenzo, 1980, 1981, 1982, 1987, 1988, 1990, 1995a, 1995b; Louis, 2003; M. del P. S. Rodríguez, 2001; Mendoza, 1988a, 1988b, 1989, 1990; MINED-CELEP, 1999; Pacheco, 1998, 2002; P. I. Pardo, 1995; Reyes, 2005; Rivero, 1999, 2001; Rivero \& Quílez, 1995a, 1995b, 2001; Roloff e cols., 1987; Ruiz, 2001; Sarduy, 1996a, 1996b; Sotomayor \& Muñoz, 1999; Tejeda, 2007; Tintorer, 1998; Tocabens, 2001; Velázquez, 2002; Y. A. Betancourt, 2005).

Se participó activamente en la creación y perfeccionamiento de la educación de escolares con necesidades educativas especiales, desde una visión optimista, desarrolladora y compensadora, inclusiva y para todos y con todos, o sea, desde la atención a la diversidad, lo que permitió, que estos escolares, tuvieran una mejor integración social, una mayor independencia y autonomía y que pudieran tener una continuidad de estudios dentro del sistema nacional de educación en las escuelas e institutos técnicos y en las universidades, como cualquier otro estudiante (Arias, 1979, 1986a, 1986b, s.n., 1988, 1989; Arias \& Llorens, 1982; Bell, 1996, 1997; Colectivo de autores 2004, 2005; J. Betancourt \& González, 2003; Rivero, 2001; Vega, 1988, 1989; Vygotski, 1989).

Se ha trabajado siempre en el asesoramiento a los programas infantiles de la televisión cubana y en la elaboración de programas de orientación y educación a la familia y a toda la población (Arias, 1999c, 2002c; Arias e cols., 1977; Cánovas-Fabelo e cols., 1997; Castillo e cols., 1989; Castro, 1996; E. Rodríguez, 2002; Febles Elejaldes, 1999, 2003; García, 1983; Ibarra, 1999; Roche, 2000; Roche \& Tocabens, 1999; Tejeda, 2007).

Se ha trabajado y se sigue trabajando por un evaluación y un diagnóstico que acabe de superar la visión psicométrica, descriptiva, clasificatoria, superficial y estigamatizante y se constituya en un diagnóstico explicativo, que oriente la intervención y la educación promotora del aprendizaje y el desarrollo infantil (Arias, 2001; García, 1983; López \& Gómez, 1996; Muzio, 1989).

Todo esto se ha logrado por la orientación que tenemos y que creemos que interpreta, lo que está en nuestra cultura y en nuestra historia, de que con el debate crítico sistemático de las teorías, de las prácticas y sus resultados, se puede lograr lo que exige, la necesidad de una aplicación práctica de los contenidos teóricos y de las investigaciones empíricas, en el mejoramiento de la Educación de un pueblo.

Todo esto se ha hecho orientado por la concepción de que las personas pueden y deben apropiarse de los 
contenidos de la educación, como condición para promover su desarrollo personal y el desarrollo sostenido de un proyecto social y cultural revolucionario. Por la necesidad de realizar estudios y sistematizaciones críticas de los contenidos teóricos, metodológicos y empíricos de toda la psicología, para construir un sistema de Educación que tenga la calidad necesaria para que se logre el aprendizaje de todos los escolares con su diversidad.

Por la importancia que tiene para una lucha contra el colonialismo cultural por lo que la psicología y los psicólogos, en conjunto con las demás ciencias afines a la Educación, tienen que promover y participar en el debate científico y técnico como base para la elaboración de las políticas públicas sobre la Educación e incidir, aun más, en el perfeccionamiento de estas políticas, para hacer mas significativo el papel de la Psicología con el compromiso social y cultural del país y sobre todo con el ser humano.

La Psicología Educacional, en Cuba, ha tenido una mayor participación en los proyectos y programas macroestructurales y en la definición, ejecución y control de las políticas públicas, en la formación continua de maestros y profesores, en el asesoramiento de los programas educacionales en general, que en un servicio más puntual y específico a los escolares con problemas, lo cual parece ser un hecho significativamente positivo de la psicología educacional y escolar cubana, en comparación con otros países.

Sin embargo, un aspecto en el que hay que seguir trabajando en Cuba, porque no se ha alcanzado lo que se podría y desea, es la atención individual de los escolares con problemas o dificultades ${ }^{1}$, por especialistas y profesionales que apoyen la labor del maestro y los profesores de la escuela general, técnica y profesional e incluso en las universidades.

Este aspecto parece que es otra diferencia con países en el mundo, donde este tipo de atención es mucho mayor, a la que se realiza en nuestro país. Este tema siempre ha estado presente en los debates y discusiones, en las proyecciones planteadas por los psicólogos educacionales del Ministerio de Educación, de las Facultades de Psicología y de los Institutos Superiores Pedagógicos, pero solo se ha logrado parcialmente en algunos subsistemas de educación.

Creo que esta manera de abordar el problema ha sido, en última instancia ventajosa, porque la atención individualizada no se necesitaría, en Cuba, para altos índices de estudiantes, dado que la calidad del sistema en general,

1 Cuando los psicólogos y pedagogos cubanos hablamos de problemas y dificultades en los escolares, por lo general, se refieren a que estos no son un producto del sujeto o se ubica la causa en el propio escolar. En la mayoría de los psicólogos e incluso, en la población en general, se conciben dichos problemas o dificultades, como una insuficiencia del sistema escolar o social-cultural, que no tiene en cuenta las exigencias del proceso educativo y las características del sujeto, para, adecuándose a ellas, ejercer una acción promotora y desarrolladora en este y evitar los problemas y dificultades. En esta concepción una atención más individualizada, forma parte de la efectividad que tiene que llegar a tener el sistema educativo, social y cultural de la sociedad, para evitar esos problemas o enmendarlos. garantiza que solo una pequeña parte de los escolares, sus maestros y sus familiares, necesiten de una atención más especializada.

No obstante, consideramos que el país tiene múltiples formas en las que se brinda esta atención más individualizada, a través de centros creados por la Federación de Mujeres Cubanas, el Centro de Educación Sexual, el sistema de Salud Pública e incluso en Centros de las Universidades, que bien pudieran ser la base de una experiencia, para crear un sólido, amplio e integral subsistema de atención individual en la Educación del país, que logre atender a la masividad y llegar a escolares y educadores con mayores dificultades.

En este sentido la experiencia que se realiza en muchos de los países de América Latina, puede ser muy útil para nosotros, de la misma manera, que nuestra experiencia en la participación, en la elaboración y mejoramiento constante de las políticas públicas, pudiera ser muy importante para esos países.

Por último, ninguna tribuna es mejor, que la que brinda este Congreso, que se promueve como genuinamente Latinoamericano, combatiente contra el colonialismo cultural de Europa y los Estados Unidos y le agregaría, si me lo permiten, cualquier otro tipo de hegemonismo, imposición y creación de ídolos; para formular estas ideas que resultan esenciales para la materialización de este discurso y para contribuir verdaderamente al desarrollo de los pueblos de nuestra Región.

La psicología como una ciencia comprometida con el ser humano, sus problemas y dificultades en el desarrollo, debe de insistir en una educación más eficiente que permita una educación para todos y de calidad, que garantice un aprendizaje eficiente en todos los escolares y con ello, el pleno desarrollo del ser humano.

Por eso proponemos que este Congreso se pronuncie con propuestas concretas y promueva con ellas, un trabajo encaminado a producir un cambio en las políticas que sobre los programas educacionales existen en la mayoría de nuestros países de América Latina.

Hace muy poco escuche en un programa sobre política en Brasil a dos senadores declarando públicamente que los programas de educación en Brasil son muy deficientes y que la mayoría de los gobernantes, históricamente, no han deseado que toda la población reciba una buena educación ¿qué podrá hacerse después de este reconocimiento? ¿Pensarán así muchos otros? ¿Podrán las ciencias psicológicas, la psicología educacional y escolar, quedarse calladas?

Pienso que aunque el capitalismo ha promovido la educación para todos desde finales del siglo XIX y XX, nunca le ha interesado, de verdad, resolver el problema de que todos aprendan y se desarrollen a los niveles que produce una buena educación. Solo les interesa resolver la fuerza de trabajo calificada que necesitan y hasta ahora la resuelven con el por ciento que logran los sistemas, por eso, pienso que se produce esta impresión en estos políticos que criticaron su sistema. Cualquier sistema que garantice que 
un $30 \%$ no aprende, que otro tanto no aprende bien y que solo se garantiza el por ciento necesario para la producción y el desarrollo económico capitalista, es bueno, ¿para qué invertir más?

Creo que se debe debatir, cómo informarles a los gobiernos que es imperdonable humanamente e inaceptable científicamente, que en los sistemas educacionales no aprendan a leer y a escribir, hasta un $30 \%$ de los escolares; cuando los retrasados mentales leves, lo pueden hacer en dos o tres cursos y los sordos ciegos, con medios pedagógicos específicos, logran hasta estudios universitarios y de posgrado de nivel de doctorado.

Que el desarrollo de la educación universitaria no se justifica, cuando no existen buenos programas de Educación infantil, inicial o preescolar y luego buenos programas de educación primaria o fundamental, o un buen vínculo y colaboración entre la familia, la escuela y la sociedad que garanticen las bases, los cimientos, de una preparación para las realizaciones de estudios medios, medios superiores y superiores.

Que hasta la UNESCO, ha tenido que reconocer, al menos declarativamente, en el discurso, que la Educación promueve el desarrollo humano y que solo con ese desarrollo es que se logrará, el desarrollo económico sostenible de los países (Delors, 1996; UNICEF, 1990).

Que para mejorar la Educación del pueblo y de la diversidad, para lograr la declarada educación inclusiva, es necesaria una voluntad política de elaborar o crear políticas públicas, orientadas a lograr una Educación de Calidad para Todos y garantizar su puesta en práctica y constante evaluación y mejoramiento.

Que es necesario promover, conjuntamente, la participación de científicos y profesionales de la pedagogía, la psicología, la psicopedagogía y otras ciencias a fines para elaborar los contenidos científicos y técnicos, para proyectar el sistema de educación que se necesita.

Que la América Latina y el mundo poseen conocimientos suficientes para proyectar mejores sistema de Educación dada la diversidad histórica de criterios y la posibilidad de síntesis crítica de estos conocimientos, lo que posiblemente nos permita mejorar lo que se ha hecho, elaborar y construir sistemas para el momento actual, relativo y no permanente, no perfecto; pero si posiblemente, más efectivos, si hacemos los análisis críticos y buscamos las mejores soluciones a nuestros problemas, con nuestros conceptos, formas de pensar y nuestras prácticas.

Pudiera mencionar otros muchos aspectos más, pero pienso que estos, ya son importantes para comenzar...

En Cuba, se han alcanzado logros, eso se encuentran en publicaciones internacionales, sin embargo, mucho más se pudiera alcanzar, por eso, la psicología educacional en Cuba no se puede vanagloriar de sus éxitos, hay que trabajar por resultados superiores y también debemos y podemos aprender de la psicología educacional de los países hermanos de la América Latina y sobre todo, colaborar de conjunto en los estudios y análisis críticos que se hagan para mejorar nuestros sistemas educacionales. Ese es un sueño que pudiera ayudar a hacerlo realidad la ULAPSI, si logra materializar lo que postula.

\section{Referencias}

Acosta, Y. I. (1998). Estudio de la incidencia, regularidades y condiciones socioambientales de factores de riesgo en niños de poblaciones socioculturales significativamente diferentes. Trabajo de Diploma, Facultad de Psicología, Universidad de la Habana, Cuba.

Arias, G. B. (1979). La Educación Especial en la República de Cuba. Em Jornada Internacional de Estimulación Precoz. España.

Arias, G. B. (1986a). La Educación Especial en Cuba. Su desarrollo y perspectivas. Em Congreso de Pedagogia - Conferencias Especiales.

Arias, G. B. (1986b). Algunas tendencias actuales sobre la educación especial. Revista Educación, 75-81.

Arias, G. B. (1988). La educación especial en Cuba. Habana: Pueblo y Educación.

Arias, G. B. (1989). Desarrollo de la Educación Especial en América Latina. Revista Maestro-Defectólogo: Problemas de La Formación.

Arias, G. B. (1999a). Acerca del valor teórico y metodológico de la obra de L. S. Vygotski. Revista Cubana de Psicología, 16(3), 171-176.

Arias, G. B. (1999b). La dinámica de lo externo y lo interno en la relación de ayuda. Psicología y Ciencia Social, 3(2), 3-10.

Arias, G. B. (1999c). La Orientación Psicológica. Un intento de elaboración teórica. En Colectivo de autores, La creación y evaluación de servicios de Orientación y Atención Psicológica. Centro de Orientación y Atención Psicológica a la Población Alfonso Bernal del Riesgo, Faculdade de Psicologia, Universidad de La Habana, Cuba.

Arias, G. B. (2001). Evaluación y Diagnóstico en la Educación y el Desarrollo. São Paulo: Laura Calejon.

Arias, G. B. (2002a). El proceso de construcción del Enfoque Histórico Cultural en sus Inicios [CD-ROM]. Em Convención HOMINIS'02. Habana: Palacio de las Convenciones.

Arias, G. B. (2002b). Las unidades funcionales en la psicología. Una tarea inconclusa y no continuada [CD-ROM]. Em Convención HOMINIS'02. Habana: Palacio de las Convenciones.

Arias, G. B. (2002c). Nuestro Psicodrama. Piracicaba, São Paulo: G.E. Degaspari.

Arias, G. B. (2002d). Por una mejor vida y aplicación del enfoque histórico cultural [CD-ROM]. Em Convención HOMINIS'02. Habana: Palacio de las Convenciones.

Arias, G. B. (2004a). Los aportes de L.S. Vygotski a la psicología de todos los tiempos. Em Ponencia a la Conferencia Internacional L.S. Vygotski. Rússia: Instituto Vygotski.

Arias, G. B. (2004b). Conferencia sobre desarrollo de la inteligencia. VI Encuentro Internacional de Educación Inicial y Preescolar Habana: Centro de Referencia Latinoamericano para la Educación Preescolar

Arias, G. B. (2005). La Persona en el Enfoque Histórico Cultural. São Paulo: Linear B.

Arias, G. B. (2006). Inteligência e Educação. São Paulo: Terceira Margem. 
Arias, G. B. (s.n.). Desarrollo Histórico de los escolares con necesidades educativas especiales. Apuntes y consideraciones. Habana: Ligeras.

Arias, G. B., e cols. (1977). La educación familiar de nuestros hijos. Habana: Pueblo y Educación.

Arias, G. B., \& Llorens V. (1982). La Educación Especial. Habana: Pueblo y Educación.

Ávila, A. A. (2002). Premisas de la actividad con los objetos en el primer año de vida. Em S. L. Lorenzo, L. A. Cao, \& A. M. S. Gómez, Estudio sobre las particularidades del desarrollo del niño preescolar cubano. Habana: Pueblo y Educación.

Bell, R. (1996). Educación Especial. Sublime Profesión de Amor. Habana: Pueblo y Educación.

Bell, R. (1997). Educación Especial: razones, visión actual y desafíos. Habana: Pueblo y Educación.

Beltrán, M. T. B. (1988a). Las relaciones entre la familia y la escuela en nuestra sociedad. Em ¿De quién es la responsabilidad: la escuela o la familia?. Habana: Pueblo y Educación.

Beltrán, M. T. B. (1988b). Las relaciones con los niños y los adolescentes $\mathrm{Em}$ ¿De quién es la responsabilidad, la escuela o la familia?. Habana: Pueblo y Educación.

Beltrán, M. T. B (1989). ¿Cómo continuar en el hogar el trabajo que realiza la escuela? Em Conoces a tus alumnos. Habana: Pueblo y Educación.

Betancourt, J. (1999). El papel de las vivencias en el proceso de formación de los trastornos del comportamiento. Tesis de Maestría, Facultad de Psicología, Universidad de La Habana, Cuba.

Betancourt, J. (2002). Vivencias en los escolares con trastornos del comportamiento y emocionales. Tesis de Doctorado, Instituto Central de Ciencias Pedagógicas, MINED, Cuba.

Betancourt, J., \& González, O. (2003). Dificultades en el aprendizaje y trastornos emocionales y de la conducta. Habana: Pueblo y Educación.

Betancourt, Y. A. (2005). Sobre Los software educativos y los niños con Trastornos de la Conducta. Dissertação de Mestrado, Faculdade de Psicologia, Universidad de La Habana, Cuba.

Bozhovich, L. I. (1976). La personalidad y su formación en la edad Infantil. Habana: Pueblo y Educación.

Calviño, M. A. (1998). Trabajar en y con grupos: Experiencias y reflexiones básicas. Habana: Academia.

Cánovas-Fabelo, L., e cols. (1997). Para la vida. Habana: Pueblo y Educación.

Castillo, S., Castro, P., \& Nuñez, E. (1989). La preparación pedagógica y psicológica de la familia. Em Ponencia Sexta reunión de investigadores de la juventud. Habana: Centro de Estudios Sociales de la Juventude.

Castro, P. L. (1996). Cómo la familia cumple su función educativa. Habana: Pueblo y Educación.

Colectivo de Autores. (2004). Psicología Especial. Tomo I. Habana: Félix Varela.

Colectivo de Autores. (2005). Psicología Especial. Tomo II. Habana: Félix Varela.

Comenio, A. J. (1983). Didáctica Magna. Habana: Pueblo y Educación.

Congreso de Educación y Cultura. (1971). Documentos del Congreso. Habana: Ministerio de Educación.

Cordero, Y. (2001). Particularidades del proceso de cambio en el desarrollo psicológico del niño y en la categoría padres potenciadores del desarrollo, al ampliar la zona de desarrollo próximo de estos, mediante un método de estimulación directa al niño e indirecta al padre. Tesis de Diploma, Facultad de Psicología, Universidad de la Habana, Cuba.

Corral, R. (1999). Las lecturas de la zona de desarrollo próximo. Revista Cubana de Psicología, 16(3), 18-26.

Cruz-Tomás, L. (1995). Acerca de la periodización del desarrollo psíquico en el niño de edad temprana. Em L. Cruz Tomás \& O. Kraftchenko (Org.), Selección de lecturas de psicología infantil y del adolescente (Tercera parte). Habana: Pueblo y Educación.

Cruz-Tomás, L., \& O. Kraftchenko. (1995). Selección de lecturas de Psicología infantil y del adolescente (Tercera Parte). Habana: Pueblo y Educación.

Cruz, C. A. (1998). Diagnóstico y zona de desarrollo próximo. Alternativa en la validación de una metódica del cuarto excluido. Tesis de Doctorado, I.C.C.P., Habana, Cuba.

Cuesta, G., \& Hernández, B. (2000). Regularidades asociadas al proceso de cambio conceptual y actitudinal en padres potenciadores y no potenciadores del desarrollo psicológico de sus hijos. Trabajo de Diploma, Faculdade de Psicologia, Universidad de la Habana, Cuba.

Davidov, V. V. (1991). La enseñanza que desarrolla. Em A. Múdrik (Org.), La educación y la enseñanza. Una mirada al futuro. Moscú: Progreso.

Dávila, M. T. (2006). ¿Qué tipos de factores de riesgo se pueden detectar en una población de niños con problemas de aprendizaje?. Trabajo de Diploma, Faculdade de Psicología, Universidad de La Habana, Cuba.

Delors, J. (1996). La educación encierra un tesoro. Madrid: UNESCO.

Días, Z. R. (2005). La orientación psicopedagógica en las familias de niños preescolares con dificultades en las habilidades previas al aprendizaje. Tesis de Maestría, Faculdade de Psicologia, Universidad de La Habana, Cuba.

Díaz, M. C., \& Rivero, M. R. (2000). La modelación espacial: una posibilidad para potenciar el desarrollo metacognitivo en el preescolar. Camagüey: CELEP.

Díaz, M. C., \& Rivero, M. R. (2001). La modelación espacial: forma de mediatización idónea en la edad preescolar. Camagüey: Artículo inédito

Domínguez, L. (1995). Algunas consideraciones acerca del problema de la periodización del desarrollo psíquico. Em L. Cruz-Tomás \& O. Kraftchenko, Selección de lecturas de psicología infantil y del adolescente (Tercera parte). Habana: Pueblo y Educación.

Esteva Boronat, M. (2002). Formación de las premisas del juego de roles en el tercer año de vida. Em S. L. Lorenzo, L. A. Cao, \& A. M. S. Gómez, Estudio sobre las particularidades del desarrollo del niño preescolar cubano. Habana: Pueblo y Educación.

Fariñas, G. L. (1995). Maestro, una estrategia para la enseñanza. Habana: Academia.

Fariñas, G. L. (1999). Acerca del concepto de vivencia en el Enfoque históricocultural. Revista Cubana de Psicología, 16(3), 222.

Fariñas, G. L. (2004a). Apuntes para una teoría compleja del aprendizaje. Em J. A. Gutiérrez, Aprendizaje y perfeccionamiento escolar (pp. 1-11). Madrid: Universidad de Alcalá de Henares.

Fariñas, G. L. (2004b). Maestro, para una didáctica del aprendes a aprender. Habana: Pueblo y Educación.

Fariñas, G. L. (2005). Psicología, Educación e, Sociedad. Habana: Félix Varela. 
Fariñas, G. L., \& Arias, G. B. (2002). L. S. Vygotski, por una Psicología General: a favor y en contra. Problemas teóricos y metodológicos [CD-ROM]. Em Convención HOMINIS'02. Habana: Palacio de las Convenciones.

Fariñas, G. L., \& Coelho, L. (2008). Cadernos Ecos - Educação, Cultura e Desenvolvimento Humano (Vol. II). São Paulo: Terceira Margem.

Febles Elejaldes, M. (1987). La Personalidad y el nivel de desarrollo del colectivo. Em Investigación de la Personalidad en Cuba. Habana: Pueblo y Educación.

Febles Elejaldes, M. (1988). Niveles de desarrollo de las intereses cognoscitivos hacia la música. Revista Cubana de Psicología, $5(2), 27-36$

Febles Elejaldes, M. (1997). Acerca de Nuestra Herencia Histórico Cultural. Revista Cubana de Psicología, 14(2), 235-239.

Febles Elejaldes, M. (1999). La Orientación Psicológica desde el Enfoque Histórico Cultural. Consideraciones generales. Em Colectivo de autores, La creación y evaluación de servicios de Orientación y Atención Psicológica. Centro de Orientación y Atención Psicológica a la Población Alfonso Bernal del Riesgo, Facultad de Psicología, Universidad de La Habana, Cuba.

Febles Elejaldes, M. (2001). La Concepción Histórico del desarrollo: Principios y leyes. Em Psicología del desarrollo. Habana: Félix Varela.

Febles Elejaldes, M. (2003). El cuerpo como mediador de las funciones psíquicas superiores: hacia una terapia corporal. Revista Cubana de Psicología, 20(3), 269-275.

Fernández, T. G. (2003). Niños de alto riesgo del barrio de Atarés. Trabaj de Diploma, Facultad de Psicología, Universidad de La Habana, Cuba.

Figueredo, H. (1999). La atención a los escolares con insuficiencias leves en el lenguaje. Tesis de Doctorado, Instituto Central de Ciencias Pedagógicas, MINED, Habana, Cuba.

Freire, P. (1971). La educación como práctica de la libertad. Madrid: Siglo XXI

Freire, P. (2001). Pedagogía de los sueños posibles. São Paulo: UNESP.

García, A. M. (1983). Selección de lecturas de evaluación y diagnóstico infantil. Habana: Andrés Voisín.

Gómez, A. M. S. (1980). La asimilación de la construcción y utilización de modelos en niños de edad prescolar. Habana: Ciencias Pedagógicas.

Gómez, A. M. S. (1988). Investigaciones psicológicas y pedagógicas acerca del niño preescolar. Habana: Pueblo y Educación.

Gómez, A. M. S. (1991). Formación y desarrollo de capacidades intelectuales. Revista Simientes, 3, p. 28.

Gómez, A. M. S. (1995). Estudio de las particularidades del desarrollo del preescolar cubano. Habana: Pueblo y Educación.

Gómez, A. M. S., \& Borgato, A. (1988). Desarrollo del pensamiento en niños de edad temprana. Solución de tareas prácticas instrumentales en niños de esta edad. Em A. M. S. Gómez \& J. H. López, Investigaciones psicológicas y pedagógicas acerca del niño preescolar. Habana: Pueblo y Educación.

González, O. (2000). Las características de los escolares con retardo en el desarrollo psíquico. Tesis de Maestría, Facultad de Psicología, Universidad de La Habana, Cuba.

Gutiérrez, J. A., López J. H., \& Arias G. B. (1990). Un Estudio del Niño Cubano. Habana: Impresora Gráfica.
Hernández, J. M. G. (2002). Aproximación al estudio de la relación entre el nivel de desarrollo real y la amplitud de la Zona de Desarrollo Próximo en dependencia de la estimulación recibida. Habana: CELEP.

Ibarra, L. M. (1993). La comunicación padres e hijos. Em ¿Nos comunicamos con nuestros hijos?. Habana: Política.

Ibarra, L. M. (1999). Una mirada a la orientación desde el enfoque Histórico-Cultural. Revista Cubana de Psicología 16(3), 77-85.

Leontiev, A. N. (1966). El concepto de reflejo. Su importancia para la Psicología Científica. Discurso inaugural del XVIII Congreso Internacional de Psicología Científica. Em Boletín de Psychologie, 254(5).

Leontiev, A. N. (1981). Actividad Conciencia y personalidad. Habana: Pueblo y Educación.

Leontiev, A. N. (1991). Prólogo al Tomo I de las Obras Escogidas. Madrid: Visor.

Leontiev, A. N. (1997). Prólogo al Tomo I de las Obras Escogidas. Madrid: Visor.

López, J. H. (1990). La solución mediatizada de tareas cognoscitivas en niños de edad prescolar. Em I Jornada Científica del ICCP. Habana: ICCP.

López, J. H (1991). Formación y desarrollo de las capacidades intelectuales. Simientes, 3, 28-33.

López, J. H. (1992). La modelación como mediatización en la solución de tareas. Em S. L. Lorenzo, L. A. Cao, \& A. M. S. Gómez, Estudio sobre las particularidades del desarrollo del niño preescolar cubano. Habana: Pueblo y Educación.

López, J. H, \& Borgato, A. (2002). Formación de la habilidad de análisis sonoro de la palabra en niños del sexto año de vida (cinco años de edad). Em S. L. Lorenzo, L. A. Cao, \& A. M. S. Gómez, Estudio sobre las particularidades del desarrollo del niño preescolar cubano. Habana: Pueblo y Educación.

López, J. H, \& Gómez, A. M. S. (1986). Dominio del arte de construir y usar modelos materiales evidentes en la infancia prescolar. Em L. A. Venguer \& J. H. López, Particularidades del desarrollo de las capacidades cognoscitivas en la edad prescolar (pp.15-25). Moscú: Vneshtorgizdat.

López, J. H, \& Gómez, A. M. S. (1996). El diagnóstico: un instrumento de trabajo pedagógico de preescolar a escolar. Habana: Pueblo y Educación.

López, J. H, e cols. (1990-2003). Conversaciones personales y debates científicos y cursos en la Cátedra L.S. Vygotski. Universidad de La Habana, Habana, Cuba.

Lorenzo, S. L. (1980). El papel de la asimilación de la planificación gráfica de la construcción en el desarrollo intelectual de los niños de edad preescolar. Tesis de Doctorado, Moscú.

Lorenzo, S. L. (1981). Las acciones internas de modelación y las capacidades intelectuales de los niños de edad prescolar. Varona $6,7,34-50$

Lorenzo, S. L. (1982). La actividad de construcción y el desarrollo intelectual en la edad prescolar. Simientes, 2, 33-38.

Lorenzo, S. L. (1987). La formación de habilidades intelectuales en el proceso de enseñanza de la construcción. Simientes, 1, 37-42.

Lorenzo, S. L. (1988). Utilización de acciones modeladoras gráficas como medio para desarrollar el pensamiento de los prescolares en el proceso de enseñanza de la actividad de construcción. Em A. M. S. Gómez \& J. H. López, Investigaciones psicológicas y pedagógicas acerca del niño preescolar (pp. 110-117). Habana: Pueblo y Educación. 
Lorenzo, S. L. (1990). Formación de las capacidades para la modelación evidente durante las clases de construcción organizadas con grupos de diferente edad en el jardín de la infancia. Em L. A. Venguer \& J. H. López, Particularidades del desarrollo de las capacidades cognoscitivas en la edad preescolar (pp. 64-71). Moscú: Vneshtorgizat.

Lorenzo, S. L. (1995a). Formación y desarrollo de las capacidades intelectuales y creativas en los juegos de construcción de los niños de edad prescolar. Em S. L. Lorenzo, L. A. Cao, \& E. Á. M., Estudio sobre las particularidades del desarrollo del niño prescolar cubano. Habana: Pueblo y Educación.

Lorenzo, S. L. (1995b). Papel de la asimilación de acciones de modelación en la actividad de dibujo: Su influencia en el desarrollo de las capacidades intelectuales y creativas de prescolares mayores. Em S. L. Lorenzo, L. A. Cao, \& A. M. S. Gómez, Estudio sobre las particularidades del desarrollo del niño prescolar cubano. Habana: Pueblo y Educación

Louis, G. P. (2003). Condiciones del desarrollo infantil y la dinámica familiar. Trabajo de Diploma, Facultad de Psicología, Universidad de La Habana, Cuba.

Luz y Caballero, J. C. de La. (1999). Obras de la Biblioteca de Clásicos Cubanos. Tomo cinco. Habana: Imagen Contemporánea.

Luz y Caballero, J. C. de La. (2001a). Obras, Escritos Educativos II. Habana: Imagen Contemporánea.

Luz y Caballero, J. C. de La. (2001b). Obras, Escritos Educativos II. Habana: Imagen Contemporánea.

Martí, J. (1975). Obras Completas. Habana: Editorial de Ciencias Sociales.

Martí, J. (1983). Otras crónicas de Nueva York. Habana: Editorial de Ciencias Sociales.

Mendoza, F. M. (1988a). La asimilación de las acciones mecánicas mediante la construcción y transformación de modelos de mecanismos simples. Em A. M. S. Gómez \& J. H. López, Investigaciones psicológicas y pedagógicas acerca del niño preescolar (pp. 91-100). Habana: Pueblo y Educación.

Mendoza, F. M. (1988b). La utilización de esquemas por los niños del quinto año de vida. En A. M. S. Gómez \& J. H. López, Investigaciones psicológicas y pedagógicas acerca del niño preescolar (pp. 74-89). Habana: Pueblo y Educación.

Mendoza, F. M. (1989). Acciones instrumentales en la edad temprana y su valor para el desarrollo intelectual. Simientes, 43.

Mendoza, F. M. (1990). Dependencia entre la formación de la actitud emocional positiva hacia las acciones con objetos y la asimilación del método de cumplirlas. Em L. A. Venguer \& J. H. López, Particularidades del desarrollo de las capacidades cognoscitivas en la edad prescolar (pp. 100-108). Moscú: Vneshtorgizdat.

MINED-CELEP. (1999). Estudio longitudinal del proceso educativo $y$ de su influencia en el desarrollo de los niños desde el 4to año de vida hasta la culminación el primer grado. Informe de Investigación. Habana.

Ministerio de Educación. (1973). III Encuentro de psicólogos que trabajan en la Educación. Habana: Ministerio de Educación Ciudad de La Habana, Cuba.

Ministerio de Educación. (1975). IV Encuentro de psicólogos que trabajan en la Educación. Habana: Ministerio de Educación Ciudad de La Habana, Cuba.

Ministerio de Educación. (1992). Educa a tu hijo. Programa para la familia dirigido al desarrollo integral del niño (Vols. 1-9). Habana: Pueblo y Educación.

Ministerio de Educación. (1994). En torno al Programa de Educación Preescolar. Habana. Pueblo y Educación.
Mitjans, A. (1991). La relación entre personalidad, motivación, creatividad. Implicaciones en la práctica educativa. Revista cubana de Psicología, 7(2-3), 104-121.

Montero, P. R. (1996). Reflexión y aprendizaje en el aula. Habana: Pueblo y Educación.

Mújina, V. S. (1981). Características psicológicas del preescolar y del preescolar. Em A. Petrovsky, Psicología Evolutiva y Pedagógica. Habana: Pueblo y Educación.

Muzio, P. A. (1989). Apuntes sobre evaluación y diagnóstico infantil. Faculdade de Psicologia, Universidad de La Habana, Cuba.

Muzio, P. A. (1990). Mi familia es así. Habana: Ciencias Sociales.

Muzio, P. A. (2002). Psicología de la Familia. Una aproximación a su estudio. Habana: Félix Varela.

Novoselova, S. (1981). El desarrollo de pensamiento en la edad temprana. Habana: Pueblo y Educación.

Pacheco, G. (1998). El problema de lo biológico y lo social en la conformación de lo psíquico: hacia un análisis histórico y dialéctico. Trabajo de Diploma, Facultad de Psicología, Universidad de La Habana, Cuba.

Pacheco, G. (2002). Función educativa de los padres y modelos de crianza en su familia de origen: un proceso de construcción individual. Tesis en opción al grado científico de Master en Psicología Educativa, Facultad de Psicología, Universidad de La Habana, Cuba.

Pardo, I. H. (2005). Orientación psicopedagógica a las familias con niños con dificultades en el aprendizaje en las edades de 6-7. Facultad de Psicología, Universidad de La Habana, Cuba.

Pardo, P. I. (1995). Caracterización de la influencia educativa en familias de condiciones Socioculturales y estructurales extremas. Trabajo de Diploma, Facultad de Psicología, Universidad de La Habana, Cuba.

Rey, F. L. G. (1989a). Personalidad y comunicación: su relación técnica y metodológica. Em Colectivo de autores, Temas sobre la actividad y la comunicación (pp. 327-347). Habana: Ciencias Sociales.

Rey, F. L. G. (1994). Comunicación Educativa y el Desarrollo de la Personalidad. Cd. México: Universidad Nacional Autónoma de México.

Rey, F. L. G. (2000). La psicología en Cuba: Un relato para su historia. Revista Interamericana de Psicología, 34(2), 57-268.

Rey, F. L. G., \& Martinez A. M. (1989). La personalidad su educación y desarrollo. Habana: Pueblo y Educación.

Reyes, O. G. (2005). Alternativa metodológica para el desarrollo de la independencia en escolares con retraso mental leve del segundo y tercer ciclo que asisten a la escuela especial. Facultad de Psicología, Universidad de La Habana, Cuba.

Rivero, M. R. (1999). Papel de los modelos gráficos en el desarrollo intelectual de retrasados mentales leves. Tesis de Maestría, ICCP, Habana, Cuba.

Rivero, M. R. (2001). Influencia de la modelación en el desarrollo intelectual de retrasados mentales leves. Tesis de Doctorado, ICCP, Habana, Cuba.

Rivero, M. R., \& Quílez, L. I. (2001). La modelación espacial: una vía para la corrección y compensación de los retrasados mentales. Artículo inédito. Camagüey.

Rivero, M. R., \& Quílez, L. I. (1995a). Influencia de la modelación en el desarrollo intelectual de escolares retrasados mentales. Trabajo investigativo. Camagüey. 
Rivero, M. R., \& Quílez, L. I. (1995b). Influencia de las acciones de modelación en el desarrollo del pensamiento de escolares retrasados mentales. Investigación. Camagüey.

Roche, O. (2000). Una aproximación a la Psicoterapia Científica desde una perspectiva histórica y dialéctica. Tesis de Maestría, Facultad de Psicología, Universidad de La Habana, Habana, Cuba.

Roche, O., \& Tocabens, B. E. (1999). El Proceso de Diagnóstico en la Orientación Psicológica. Em Colectivo de autores, La creación y evaluación de servicios de Orientación y Atención Psicológica. Centro de Orientación y Atención Psicológica a la Población Alfonso Bernal del Riesgo, Facultad de Psicología, Universidad de La Habana, Habana, Cuba.

Rodríguez, E. (2002). Diferencias individuales. Módulo de maestría de Psicología Educativa, Faculdade de Psicologia, Universidad de La Habana, Cuba.

Rodríguez, M. del P. S. (2001). Los educadores potenciadores del desarrollo infantil. Sus cualidades profesionales y personales. Tesis de Maestría, CELEP, Habana, Cuba.

Roloff, G. e cols. (1987). Investigaciones psicodagógicas acerca del escolar cubano. Habana: Pueblo y Educación.

Rousseau, J. J. (1973). Emilio, o La Educación en Obras Escogidas. Habana: Editorial Ciencias Sociales.

Rubinstein, S. L. (1965). El ser y la conciencia. Habana: Universitaria.

Rubinstein, S. L. (1972). Principio de Psicología General. Habana: Revolucionaria.

Ruiz, D. (2001). La categoría Zona de Desarrollo Próximo como método para la optimización del proceso de potenciación del desarrollo infantil. Trabalho de Diploma, Faculdade de Psicología, Universidad de La Habana, Habana, Cuba.

Sarduy, A. L. (1996a). Interacción en zona de desarrollo próximo. Qué puede para bien y qué para mal. Habana: ICCP-ARGOS.

Sarduy, A. L. (1996b). Pensamiento, análisis y autorregulación de la actividad cognitiva de los alumnos. Habana: Pueblo y Educación.

Serra, D. J. G. (2003). Martí, Vygotski y el carácter sociohistórico del ser humano. Revista Cubana de Psicología, 20(3), 256-259.

Sotomayor, I., \& Muñoz, N. (1999). Relación entre el proceso de estimulación de la familia y los niveles de funcionamiento psicológico en niños de alto riesgo en edad temprana y preescolar. Trabajo de Diploma, Facultad de Psicología, Universidad de La Habana, Cuba.

Tejeda, C. G. (2007). El papel de la familia en la potenciación del desarrollo infantil. Una propuesta para su fortalecimiento. Trabalho de diploma, Faculdade de Psicologia, Universidad de la Habana, Habana, Cuba

Tintorer, T. (1998). Promoción de niveles superiores de funcionamiento psicológico en niño alto riesgo: una propuesta metodológica en pos del mejoramiento de la labor educativa de los padres. Tesis, Facultad de Psicología, Universidad de La Habana, Cuba.

Tocabens, B. E. (2001). Los niveles de ayuda en la relación terapéutica. Un análisis para su comprensión desde el enfoque histórico-cultural. Tesis de Maestría, Facultad de Psicología, Universidad de La Habana, Cuba.

UNICEF. (1990). Declaración Mundial sobre la Educación para Todos y Marco de Acción para Satisfacer las Necesidades Básicas de Aprendizaje. Conferencia Mundial sobre Educación para Todos. New York: Comisión Interangencial (PNUD; UNESCO; UNICEF; BANCO MUNDIAL).
Valdés, J. J. G. (1984). Apuntes para una Historia de la Psicología en Cuba. Obra inédita.

Varela, F. (1999). Ética y Anticipación del Pensamiento de la Emancipación Cubana. Habana: Imagen Contemporánea.

Vega, R. V. (1988). Trastornos psicológicos del niño y del adolescente. Faculdade de Psicologia, Universidad de la Habana, Habana, Cuba.

Vega, R. V. (1989). Psicoterapia Infantil. Habana: Pueblo y Educación.

Velázquez, P. (2002). Desarrollo intelectual en niños de alto riesgo en el primer grado. Tesis de Diploma, Facultad de Psicología, Universidad de la Habana, Habana, Cuba.

Venguer, L. A. (1976). Temas de psicología preescolar. Habana: Científico-Técnica.

Venguer, L. A., \& López, J. H. (1990). Desarrollo de las capacidades cognoscitivas del prescolar como procedimiento para dominar las formas mediatas del conocimiento. Em L. A. Venguer \& J. H. López, Particularidades del desarrollo de las capacidades cognoscitivas en la edad prescolar (pp 5-14). Moscú: Vneshtorgizdat.

Vives, J. L. (1997). Las disciplinas. Valencia, España: Artes Gráficas Soler.

Vygodskaia, G. (1997). Discurso de Gita L. Vygodskaia con motivo de la apertura del $19^{\circ}$ Coloquio de la Sociedad Internacional de la Psicología Escolar (ISPA). Revista Cuadernos Educación UCAB, 1.

Vygotski, L. S. (1996). Obras Escogidas. Tomo IV. Madrid: Aprendizaje/Visor.

Vygotski, L. S. (1972). Psicología del Arte. Barcelona, España: Breve Biblioteca de Reforma Barral.

Vygotski, L. S. (1981). Pensamiento y lenguaje. Habana: Edición Revolucionaria.

Vygotski, L. S. (1987). Historia del desarrollo de las funciones psíquicas superiores. Habana: Científico Técnica.

Vygotski, L. S. (1988). Interacción entre enseñanza y desarrollo. Em Colectivo de Autores, Selección de Lecturas de Psicología de las Edades I. Habana: Editorial del Ministerio de Educación Superior.

Vygotski, L. S. (1989). Fundamentos de Defectología. Obras Completas. Habana: Pueblo y Educación.

Vygotski, L. S. (1991). Obras Escogidas. Tomo I. Madrid: Aprendizaje/ Visor.

Vygotski, L. S. (1993). Obras Escogidas. Tomo II. Madrid: Aprendizaje/Visor.

Vygotski, L. S. (1995). Obras Escogidas. Tomo III. Madrid: Aprendizaje/Visor.

Vygotski, L. S. (1999). Imaginación y creación en la edad infantil.

(2a ed.). Habana: Pueblo y Educación.

Vygotski, L. S. (2001). Psicología Pedagógica. Un curso breve. Buenos Aires: Aique.

Vygotski, L. S. (2004). Teoría de las Emociones. Estudio históricopsicológico. Madrid: Akal. 
Recebido em: 24/05/2009

Aceito em: 25/07/2009

Sobre o Autor

Guillermo Arias Beatón

Professor Titular da Facultad de Psicología

Universidad de La Habana

Bolsista CAPES/USP 2009 